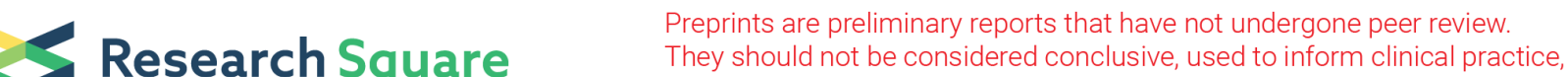 or referenced by the media as validated information.
}

\section{Early Epidemiological Features and Trends of the COVID-19 Outbreak in Southeast Asia: a Population- Level Observational Study}

\section{Mingjian Zhu}

Zhejiang University First Affiliated Hospital State Key Laboratory for Diagnosis and Treatment of Infectious Diseases

\section{Kleepbua Jirapat}

Zhejiang University School of Medicine

\section{Jian Shen}

Zhejiang University First Affiliated Hospital State Key Laboratory for Diagnosis and Treatment of Infectious Diseases

\section{Sien Ping Chew}

Shanghai Jiao Tong University School of Medicine

\section{Joanna Wei Hui Tan}

National University Singapore Faculty of Arts and Social Sciences

\section{Natthjija Latthitham}

Zhejiang University School of Medicine

\section{Lanjuan Li ( $\square$ ljli@zju.edu.cn )}

Zhejiang University First Affiliated Hospital State Key Laboratory for Diagnosis and Treatment of Infectious Diseases https://orcid.org/0000-0002-2861-2354

\section{Research article}

Keywords: COVID-19, epidemiological characteristics, trend, Southeast Asia

Posted Date: September 4th, 2020

DOl: https://doi.org/10.21203/rs.3.rs-34707/v2

License: (c) (i) This work is licensed under a Creative Commons Attribution 4.0 International License. Read Full License 


\section{Abstract}

Background: The global outbreak of coronavirus disease 2019 (COVID-19) has been ongoing in Southeast Asia since 13 January 2020. We conducted an observational study to investigate underlying disease patterns of COVID-19 in Southeast Asia, and consequently to guide intervention strategies against the pandemic.

Methods: In this population-level observational study set in Southeast Asia, we compiled a list of patients with COVID-19 $(n=925)$ and daily country-level case counts $(n=1346)$ from 13 January 2020 through 16 March 2020. All epidemiological data were extracted from official websites of the WHO and health authorities of each Southeast Asian country. Relevant spatiotemporal distributions, demographic characteristics, and short-term trends were assessed.

Results: A total of 1,346 confirmed cases of COVID-19, with 217 (16.1\%) recoveries and 18 (1.3\%) deaths, were reported in Southeast Asia as of 16 March 2020. Early transmission dynamics were examined with an exponential regression model: $y=0.30 e^{0.13 x}\left(p<0 \cdot 01\right.$, adjusted $\left.R^{2}=0.96\right)$. Using this model, we predicted that the cumulative number of reported COVID-19 cases in Southeast Asia would exceed 10,000 by early April 2020. A total of 74 cities across eight countries in Southeast Asia were affected by COVID19. Most of the confirmed cases were located in five international metropolitan areas. Demographic analyses of the 925 confirmed cases indicated a median age of 44 years and a sex ratio of 1.25 . The median age of the local patient population was significantly higher than that of the corresponding country's general population $(p<0 \cdot 01)$, whereas the sex ratio did not significantly differ.

Conclusions: The COVID-19 situation in Southeast Asia is unevenly geographically distributed and pessimistic in the short term. Age may play a significant role in both the susceptibility to and outcome of infection. Real-time active surveillance and targeted intervention strategies are urgently needed to contain the pandemic.

\section{Background}

An unknown infectious disease caused by severe acute respiratory syndrome coronavirus 2 (SARS-CoV-2) emerged in Wuhan, China, in December 2019 [1]. The disease was later officially named coronavirus disease 2019 (COVID-19). The World Health Organization (WHO) declared COVID-19 a pandemic on 11 March 2020 due to its rapid global spread [2].

The global outbreak of COVID-19 has been ongoing in Southeast Asia since 13 January 2020, making Southeast Asia the first affected region outside of China. Southeast Asia (SE Asia) consists of 11 countries: Brunei, Cambodia, Indonesia, Laos, Malaysia, Myanmar, the Philippines, Singapore, Thailand, Timor-Leste, and Vietnam. As a regional unit, it not only borders China but also lies at "the crossroads of the world" due to important maritime trade routes. In the context of globalization, regional disease surveillance is essential because it contributes to the formulation of responses to such emerging infectious diseases [3]. There have been many early epidemiological analyses of COVID-19 outbreaks in 
different countries, but none for Southeast Asia as a whole. The purpose of this observational study is to investigate the underlying disease patterns of COVID-19 in this region, and consequently to guide pandemic intervention strategies.

\section{Methods}

\section{Study Design}

In this population-level observational study, we performed a retrospective analysis of COVID-19 early epidemiological data from all 11 countries in Southeast Asia during the period between 13 January 2020 and 16 March 2020. Primary data sources were the official websites of the WHO and the public health authorities (such as the Ministries of Health or Centers for Disease Control) in relevant countries. We included individuals with a positive polymerase chain reaction (PCR) test for SARS-CoV-2 $(n=1346)$. An Excel spreadsheet database was created through data compilation and used for analyses. First, we illustrated the temporal and spatial distributions of the COVID-19 outbreak in Southeast Asia. Then, shortterm predictions about cumulative case counts were made based on the temporal distributions. Finally, we described the demographic characteristics of confirmed patients and compared them with the corresponding country's general population.

\section{Data Compilation}

We closely monitored updates from press releases and situation reports on COVID-19 issued by each Southeast Asian country's health authorities and the WHO between 13 January 2020 and 16 March 2020. Using a structured information form, our multilingual team directly and in real-time extracted epidemiological data that included daily case counts, outbreak maps, and basic demographic characteristics such as age, sex and nationality. We only compiled individual-level data for 925 cases that tested positive for COVID-19 since health authorities in Malaysia and Indonesia did not disclose relevant details since 13 March 2020, which resulted in a reduction in the sample size for demographic analysis. The median ages and sex ratios of the general populations were extracted from World Population Prospects 2019 of the United Nations and World Bank Open Data. After cross-checking, all extracted data were entered into an Excel spreadsheet database for further quantitative analysis.

\section{Statistical Analysis}

Descriptive statistical methods were used to analyze the spatiotemporal and population distributions of COVID-19 in Southeast Asia. An epidemic curve and semi-logarithmic line graph were constructed by the report date. The spatial distribution of confirmed cases was illustrated with marked maps. We also assessed the age, sex, and nationality of individuals with COVID-19 and those who died of it. Demographic data were expressed as median (interquartile range, IQR) or $n(\%)$, as appropriate. Crude recovery or fatality rates were calculated based on the reported cumulative counts. 
Paired t-tests and Mann-Whitney $\mathrm{U}$ tests were used to compare median ages and sex ratios between patients and general populations, as well as to make comparisons between deceased and surviving cases. An exponential regression model was constructed to estimate short-term incidence trends, and we subsequently tested its statistical significance and goodness-of-fit.

Hypothesis testing, analyses, and model building were performed using SPSS statistical software version 26.0 (IBM Corp., Armonk, NY, USA). The spatial distribution was mapped using R software version 3.6.2 (R Foundation for Statistical Computing). A $P$-value $<0.05$ was considered to be statistically significant.

\section{Ethical Approval}

The ethical approval or individual consent was not applicable.

\section{Results}

As of 16 March 2020, 1,346 confirmed cases of COVID-19 were reported in Southeast Asia. Of these, 217 patients recovered, and 18 patients died. The crude recovery and fatality rates were $16.1 \%$ and $1.3 \%$, respectively.

\section{Temporal Distribution}

An epidemic curve of confirmed cases (by report date) indicated that there were two distinct phases: (1) 13-29 February 2020 (first phase) and (2) 1-16 March 2020 (second phase). Cases of COVID-19 during the first phase of infections were relatively mild, with only a few confirmed cases reported daily, and most were from Singapore and Thailand. However, in the second phase, the daily reported numbers of confirmed cases increased rapidly, especially in Malaysia. The highest jump in new COVID-19 infections was recorded in Malaysia on 15 March 2020, with a single-day increase of 190 new cases (Figure 1).

A semi-logarithmic line graph of the distribution of cumulative cases over time revealed that the transmission rate of COVID-19 in Southeast Asia significantly increased at the population level at the beginning of March 2020. In contrast, the transmission rate in China remained stable (Figure 2).

An exponential curve was used to characterize the temporal distribution of cumulatively reported cases in the second phase. We obtained the following regression model: $y=0.30 e^{0.13 x}(y$ is the cumulative number of confirmed cases in the second phase and $x$ is the number of days from the first reported case in Southeast Asia). Analysis of variance (ANOVA) indicated that this model was statistically significant $(F=355.48, p<0.01)$, and the adjusted $R^{2}=0.96$. According to the model, the cumulative number of confirmed cases of COVID-19 in Southeast Asia was predicted to exceed 10,000 by early April 2020 (Figure 3).

\section{Spatial Distribution}


By 16 March 2020, eight countries in Southeast Asia (except Laos, Myanmar and Timor-Leste) reported confirmed cases of COVID-19. Malaysia ( $n=553)$, Singapore $(n=243)$, and Thailand $(n=147)$ reported the highest numbers of COVID-19 infections, accounting for $70.1 \%$ of the total cases reported in Southeast Asia. Notably, Singapore had the highest number of recovered cases $(n=109)$ with a crude recovery rate of $44.9 \%$. The most deaths occurred in the Philippines $(n=12)$ and Indonesia $(n=5)$ with crude fatality rates of $8.5 \%$ and $3.7 \%$, respectively.

In the first phase, $69.3 \%$ of the confirmed COVID-19 cases were primarily concentrated in two major international metropolises (Singapore and Bangkok) (Figure 4A). Onset focus areas of COVID-19 infections expanded to other international metropolises in this region, including Manila, Kuala Lumpur and Jakarta. The number of affected cities rose to 74, giving the pandemic a "cancer metastasis-like" spatial distribution, especially in the Malay Peninsula (Figure 4B).

\section{Demographic Characteristics}

The sample size for the demographic analysis was 925 . Of these, the age of one patient from Cambodia and the sex of one patient from Indonesia were unknown because the health authorities in Cambodia and Indonesia did not publish this information. Moreover, 104 cases from Malaysia were missing values for age; these data were imputed with a stochastic simulation method based on the age distribution of confirmed cases as of 13 March 2020 issued by the Ministry of Health, Malaysia [4]. Table 1 summarizes the demographic characteristics of confirmed COVID-19 cases.

Table 1. Basic demographic characteristics of the study population. ${ }^{\text {a }}$

\begin{tabular}{lll}
\hline & Confirmed cases (n=925) & Deceased cases (n=18) \\
\hline Age, years & $44(32-58)$ & $58(51-67)$ \\
Sex & & \\
Male & $514(56 \%)$ & $11(61 \%)$ \\
Female & $410(44 \%)$ & $7(39 \%)$ \\
Nationality & & \\
Local & $750(81 \%)$ & $15(83 \%)$ \\
Foreign & $175(19 \%)$ & $3(17 \%)$ \\
Underlying conditions & N/A & $13(72 \%)$ \\
\hline
\end{tabular}

a. Data are median (IQR) or n (\%).

Demographic analysis revealed that COVID-19 patients were primarily aged 20-69 years. This age group constituted $88.9 \%$ of the total confirmed cases in Southeast Asia. The proportion of COVID-19 cases among individuals aged $\geq 60$ years was $21.9 \%$ (Figure 5 ). 
The ages of individuals with COVID-19 in Southeast Asia ranged from 0.25-96 years, with a median age of 44 years. There were 514 males and 410 females, with a sex ratio of 1.25. The median ages and sex ratios for populations with confirmed COVID-19 cases (PWCC, both overall and local) and the general population (GP) in each country are presented in Figure 6A and Figure 6B, respectively. Moreover, the median age of PWCC (local nationals) was significantly higher than that of the corresponding GP (paired t-test; $p<0.01$ ), whereas the sex ratio did not significantly differ between the two population groups (paired t-test; $p>0 \cdot 05$ ).

Confirmed COVID-19 cases in Southeast Asia were predominantly local cases (81.1\%). Cases among foreign nationals were chiefly from China (7.2\%), Europe (5.0\%) and other Asian countries (4.4\%). Excluding those countries with a total case count $<50$, Vietnam had the highest proportion of COVID-19 cases among foreign nationals (34.4\%), whereas Brunei had the least (1.9\%).

The median age of the 18 COVID-19 related deaths recorded during the study period was 58 years, which was significantly higher than that of surviving cases (43 years) (Mann-Whitney U Test; $p<0 \cdot 01$ ). Thirteen (72.2\%) patients who died from COVID-19 infection had underlying conditions prior to the diagnosis of COVID-19. Of these, the most common underlying conditions were diabetes and/or chronic cardiovascular diseases (present in 10 cases). Dengue fever, asthma and kidney transplantation were the underlying conditions present in the other three cases that died.

\section{Discussion}

This study retrospectively analyzed early population-level data for the COVID-19 outbreak in Southeast Asia. Relevant spatiotemporal distributions and demographic characteristics were described for the first time. In addition, a predictive model was successfully constructed to estimate short-term incidence trends.

Epidemic curve and semi-logarithmic line graphs consistently illustrated two distinct phases in the epidemic. The second phase began at the start of March 2020, and was characterized by a substantial increase in the number of reported cases. The sudden increase in confirmed COVID-19 cases was a consequence of mass gatherings for various events such as Sri Petaling tabligh (a Muslim religious gathering), which triggered cluster outbreaks in Malaysia [4]. It indicated that COVID-19 was entering a rapid transmission phase. WHO classified five countries in Southeast Asia (Indonesia, Malaysia, Singapore, Thailand and Vietnam) as countries with local transmission on 2 March 2020 [5], and later declared the outbreak a pandemic on 11 March 2020 [2].

Epidemics typically follow the law of exponential growth in their early stages, especially for infectious diseases with a basic reproduction number $\left(\boldsymbol{R}_{\mathbf{0}}\right)>1$.0. The $\boldsymbol{R}_{\mathbf{0}}$ for COVID-19 was estimated to be approximately 2.2 in a previous study that focused on early transmission dynamics in Wuhan, China [6]. Our study observed a similar exponential growth trend, which was applied to the prediction of a shortterm incidence trend for COVID-19 within Southeast Asia. The goodness-of-fit (adjusted $\mathrm{R}^{2}$ ) of the 
prediction model within the second phase of transmission was 0.96 . Although the exponential growth model also well fit the early epidemic patterns of COVID-19 in some other regions like Europe and Africa, the estimates of the relevant parameters in the model varied from each other. This phenomenon could be related to different climatic conditions, genetic background and sufficiency of health resources (especially detection capabilities) $[7,8]$. Our model predicted that the cumulative COVID-19 cases in Southeast Asia would exceed 10,000 by early April 2020. In actuality, the cumulative number of COVID-19 patients in Southeast Asia was 10,153 as of 1 April 2020. This actual figure validates our prediction. Despite the effectiveness of short-term forecasts, we observed that actual case counts reported since Day 85 (6 April 2020) have gradually exceeded the lower limit of prediction. It is worth noting that Malaysia, which was the hardest hit in Southeast Asia at that time, implemented the Movement Control Order (MCO) nationwide on 18 March 2020 [9]. Thus, we speculate that the implementation of more stringent precautions may be a vital reason for the slowdown in cumulative case growth.

A geo-temporal map illustrated the spatial distribution of confirmed cases, which presented a condition similar to that of "cancer metastasis." Most of the confirmed COVID-19 cases were concentrated within several international metropolitan areas before spreading to other small-to-medium-sized cities. Several studies have demonstrated the link between transportation and the spread of COVID-19 infections. This seems to explain why metropolises, which are major international transportation hubs, were more vulnerable to an increased concentration of COVID-19 cases $[10,11]$. The smaller concentration of COVID19 cases in small-to-medium sized cities may also be partially explained by the "Iceberg Phenomenon" of disease, which indicates that infections occurring in small-to-medium sized cities are often not diagnosed in a timely manner due to a lack of laboratory testing (especially in the early stages of an outbreak). Thus, subsequent increases in the number of confirmed cases in these areas might be partly due to improvement in testing access, not merely the spread from metropolises.

We were not able to directly compare the distribution of age or sex within patient groups from different countries or regions because of considerable differences in population structure. However, we determined that the median age of locally infected COVID-19 patients was significantly higher than that of their respective country's general populations, which indicates that age may be a significant risk factor for COVID-19. This reinforces the previous finding that COVID-19 seems to be uncommon in children $[12,13]$. In terms of sex, the reported proportion of males in confirmed COVID-19 cases in China, Italy and South Korea was $51.4 \%, 59.8 \%$ and $37.7 \%$, respectively [14-16]. Although more than half of the confirmed cases in Southeast Asia were male, the link between sex and COVID-19 susceptibility was not supported by the result of our corresponding hypothesis test. Moreover, participation in social activities may be considered an intermediary factor $[12,16]$. Interestingly, the age and sex composition of confirmed cases in Vietnam were unique in that many more young and female individuals were infected with COVID-19. This may be attributed to the population structure and the role of women in Vietnamese society. Nearly $20 \%$ of confirmed COVID-19 cases in Southeast Asia included foreign nationals, but this proportion varied between countries. Diversity in the composition of patients' nationalities may suggest to some extent the risk of virus inputs. As an active center of the global community, frequent cross-border population 
movements increased human-to-human transmission within Southeast Asia [17]. Consequently, there is a need to give serious consideration to the rapid spread of the epidemic outside of China.

The global crude fatality rate for COVID-19 was $3.9 \%$ (6,606 deaths out of 167,515 confirmed cases) as of 16 March 2020 (the end of our study period) [18]. The crude fatality rate for Southeast Asia was largely underestimated in this study because of delayed diagnosis and lack of transparency in the information given by health authorities. For example, the number of deaths from COVID-19 in Indonesia jumped from five on 16 March 2020 to 19 on 18 March 2020, thus increasing the crude fatality rate to $8.4 \%$ [19]. Although most COVID-19 patients may exhibit mild clinical symptoms, older people and individuals with underlying medical conditions may be at increased risk of suffering severe illness and death. Our study results are consistent with this finding $[14,16]$. The median age of the 18 deaths included in the study was significantly higher than that of surviving cases. Among COVID-19 patients who died, $72.2 \%$ had underlying conditions, such as diabetes and chronic cardiovascular diseases, among others. Notably, one deceased case from Thailand also had dengue fever [20], a tropical disease that is common and active in Southeast Asia. It is difficult to distinguish these two viral diseases since they share some clinical and laboratory features. Public health security in this region is facing unprecedented challenges [21].

Despite our efforts to ensure data quality and analytical rigor, the present study has several limitations. First, raw data were compiled from publicly available information, which was not equally available across the countries included in the study. Therefore, the data available for the overall analysis and sample size for demographic analysis were limited. Moreover, due to the delay in the diagnosis of COVID-19 infections

and lack of transparency in the provided information, the number of COVID-19 cases and deaths may not have been comprehensively reported during the early phase of transmission. This may have resulted in an underestimation of the true severity of the outbreak in this region. Finally, evolving health policies and opportunistic factors make it difficult to predict pandemic trends. We used a simple and practical model to make short-term predictions of COVID-19 incidence trends in the study region.

\section{Conclusions}

This study was the first to describe the early epidemiological features and trends of the COVID-19 outbreak in Southeast Asia from a regional perspective. Analysis of spatiotemporal distribution characteristics indicated that the region's COVID-19 situation was unevenly geographically distributed and pessimistic in the short term. Advanced age may play a significant role in increasing susceptibility to COVID-19 infection and lead to severe clinical outcomes. Consequently, there is an urgent need to implement real-time active surveillance and develop targeted intervention strategies for combatting the pandemic.

\section{List Of Abbreviations}

SARS-CoV-2: severe acute respiratory syndrome coronavirus 2 
COVID-19: coronavirus disease 2019

WHO: the World Health Organization

SE Asia: Southeast Asia

PCR: polymerase chain reaction

IQR: interquartile range

ANOVA: analysis of variance

GP: general population

PWCC: population with confirmed COVID-19 cases

\section{Declarations}

\section{Ethics approval and consent to participate}

All data in this study were derived from the information publicly disclosed by officials and involved no individual privacy; hence, the need for ethics approval was waived by the Ethics Committee of The First Affiliated Hospital of Medical College, Zhejiang University.

\section{Consent for publication}

Not applicable.

\section{Availability of data and materials}

The datasets used and/or analyzed during the current study are available from the corresponding author on reasonable request.

\section{Competing interests}

The authors declare that they have no competing interests.

\section{Funding}

This work was supported by the National Natural Science Foundation of China [81790631, 81570512] and the National Key Research and Development Program of China [2018YFC2000500]. However, all of the funders had no role in the design, analysis, preparation of the manuscript, or decision to publish.

\section{Authors' contributions}


MZ and LL had the original idea and developed the study protocol. MZ, KJ, SPC, JWHT and NL were involved with the data collection and collation. MZ, JS, KJ and LL performed the analysis and drafted the initial manuscript. All authors contributed in the preparation of manuscript writing, and approved its final version.

\section{Acknowledgements}

Not applicable.

\section{References}

1. Zhou P, Yang XL, Wang XG, et al. A pneumonia outbreak associated with a new coronavirus of probable bat origin. Nature 2020;579:270-3.

2. WHO. Statement on the second meeting of the International Health Regulations (2005) Emergency Committee regarding the outbreak of novel coronavirus (2019-nCoV). Geneva: World Health Organization, 2020. https://www.who.int/news-room/detail/30-01-2020-statement-on-the-secondmeeting-of-the-international-health-regulations-(2005)-emergency-committee-regarding-the-outbreakof-novel-coronavirus-(2019-ncov) (accessed 10 Feb, 2020).

3. Bond KC, Macfarlane SB, Burke C, Ungchusak K, Wibulpolprasert S. The evolution and expansion of regional disease surveillance networks and their role in mitigating the threat of infectious disease outbreaks. Emerg Health Threats J 2013;6:19913.

4. KPK Press Statement 13 March 2020 - Current Situation of Coronavirus Disease 2019 (COVID-19) in Malaysia. Director-General of Health Malaysia,13 March 2020.https://kpkesihatan.com/2020/03/13/kenyataan-akhbar-kpk-13-mac-2020-situasi-semasajangkitan-penyakit-coronavirus-2019-covid-19-di-malaysia/ (accessed 13 Mar, 2020; in Malay).

5. WHO. Coronavirus disease (COVID-2019) situation reports-42. Geneva: World Health Organization, 2 March 2020. https://www.who.int/emergencies/diseases/novel-coronavirus-2019/situation-reports (accessed 2 Mar, 2020).

6. Li Q, Guan X, Wu P, et al. Early transmission dynamics in Wuhan, China, of novel coronavirus-infected pneumonia. N Engl J Med 2020;13:1199-1207.

7. Yuan J, Li M, Lv G, Lu ZK. Monitoring transmissibility and mortality of COVID-19 in Europe. Int J Infect Dis 2020;95:311-315.

8. Adegboye OA, Adekunle Al, Gayawan E. Early Transmission Dynamics of Novel Coronavirus (COVID19) in Nigeria. Int J Environ Res Public Health 2020;17:9.

9. KPK Press Statement 18 March 2020 - Current Situation of Coronavirus Disease 2019 (COVID-19) in Malaysia. Director-General of Health Malaysia,18 March 2020.https://kpkesihatan.com/2020/03/18/kenyataan-akhbar-kpk-18-mac-2020-situasi-semasajangkitan-penyakit-coronavirus-2019-covid-19-di-malaysia/ (accessed 18 Mar, 2020; in Malay). 
10. Chinazzi, M., Davis JT, Ajelli M, et al. The effect of travel restrictions on the spread of the 2019 novel coronavirus (COVID-19) outbreak. Science 2020;368:395-400.

11. Zhao S., Zhuang Z, Ran J, et al. The association between domestic train transportation and novel coronavirus (2019-nCoV) outbreak in China from 2019 to 2020: A data-driven correlational report. Travel Med Infect Dis 2020;33:101568.

12. Sun K, Chen J, Viboud C. Early epidemiological analysis of the coronavirus disease 2019 outbreak based on crowdsourced data: a population-level observational study. Lancet Digit Health 2020;2:e201-8.

13. Lee PI, Hu YL, Chen PY, Huang YC, Hsueh PR. Are children less susceptible to COVID-19? J Microbiol Immunol Infect 2020; doi: 10.1016/j.jmii.2020.02.011.

14. The Novel Coronavirus Pneumonia Emergency Response Epidemiology Team. The epidemiological characteristics of an outbreak of 2019 novel coronavirus diseases (COVID-19) in China. CCDC Weekly 2020;2:113-22.

15. Livingston E, Bucher K. Coronavirus disease 2019 (COVID-19) in Italy. JAMA 2020;323:1335.

16. Yoo JH, Chung MS, Kim JY, et al. Report on the epidemiological features of Coronavirus disease 2019 (COVID-19) outbreak in the Republic of Korea from January 19 to March 2, 2020. J Korean Med Sci 2020;35:e112.

17. Wang Y, Wang Y, Chen Y, Qin Q. Unique epidemiological and clinical features of the emerging 2019 novel coronavirus pneumonia (COVID-19) implicate special control measures. J Med Virol 2020;92:568-76.

18. WHO. Coronavirus disease (COVID-2019) situation reports-56. Geneva: World Health Organization, 16 March 2020. https://www.who.int/emergencies/diseases/novel-coronavirus-2019/situation-reports (accessed 16 Mar, 2020).

19. Kemenkes RI. Current situation of Coronavirus disease 2019 (COVID-19) in Indonesia. Ministry of Health Indonesia, 18 March 2020.https://covid19.kemkes.go.id/situasi-infeksi-emerging/info-coronavirus/situasi-terkini-perkembangan-coronavirus-disease-covid-19-18-march-2020/(accessed 18 Mar, 2020; in Indonesian).

20. MOPH. Report COVID-19 situation in Thailand. Ministry of Public Health Thailand, 1 March 2020. https://pr.moph.go.th/?url=pr/detail/2/04/139295 (accessed 1 Mar, 2020; in Thai).

21. Yan, G., Lee CK, Lam LTM, et al. Covert COVID-19 and false-positive dengue serology in Singapore. Lancet Infect Dis 2020;20:536.

\section{Figures}




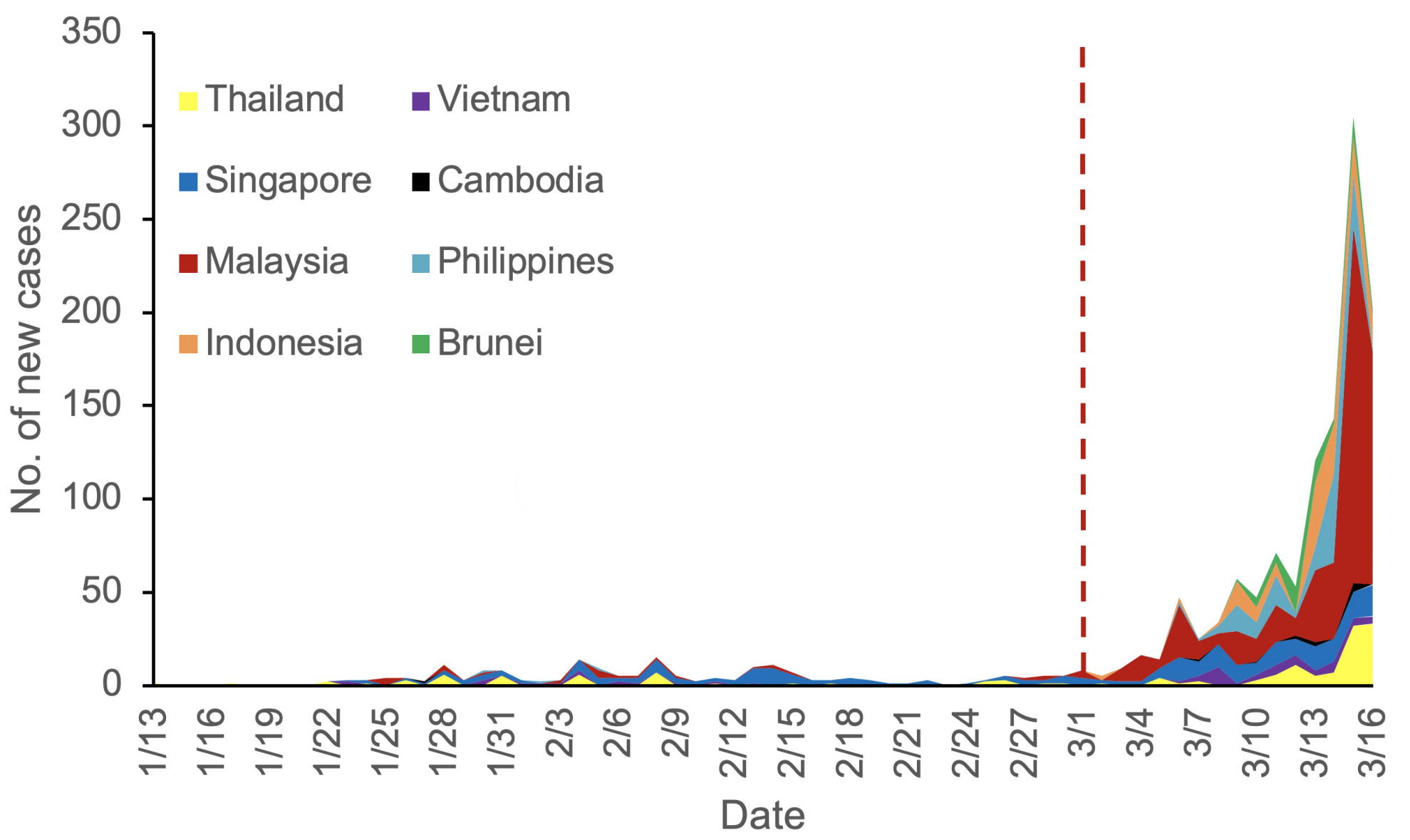

Figure 1

Epidemic curve of confirmed COVID-19 in Southeast Asia, by date of report and country from 13 January to 16 March 2020. Red dashed line: watershed (on 1 March) between the first and second phases. 


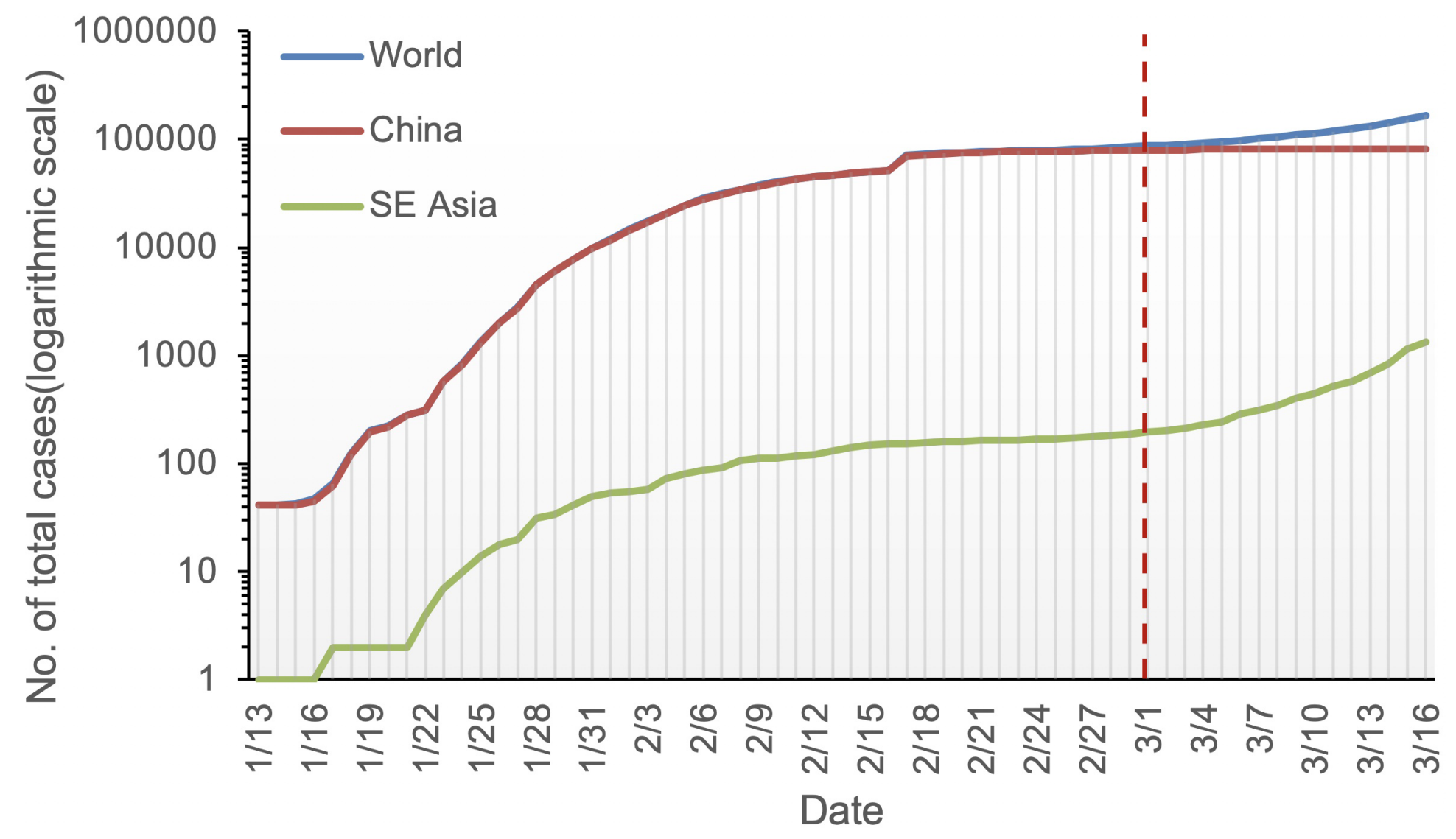

Figure 2

Semi-log graph showing the change in total cases from 13 January to 16 March 2020 for the World, China and Southeast Asia (SE Asia). Red dashed line: watershed (on 1 March) between the first and second phases. 


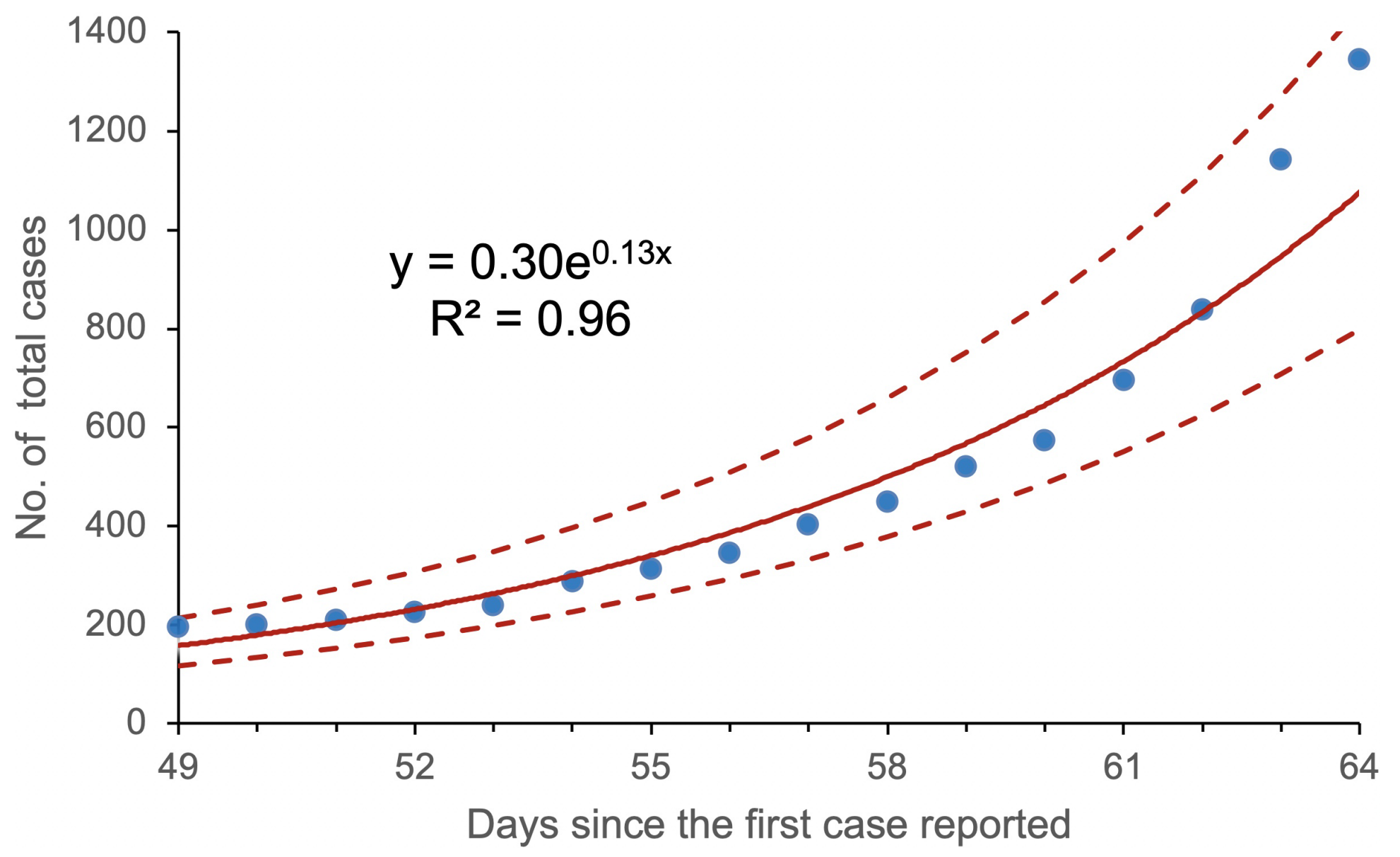

Figure 3

Exponential regression model fitting for the temporal distribution law of total cases in Southeast Asia since 1 March 2020 (the second phase). Blue dots: actual distribution of values; red solid line: exponential regression curve; red dashed lines: upper and lower limits of 95\% confidence interval.

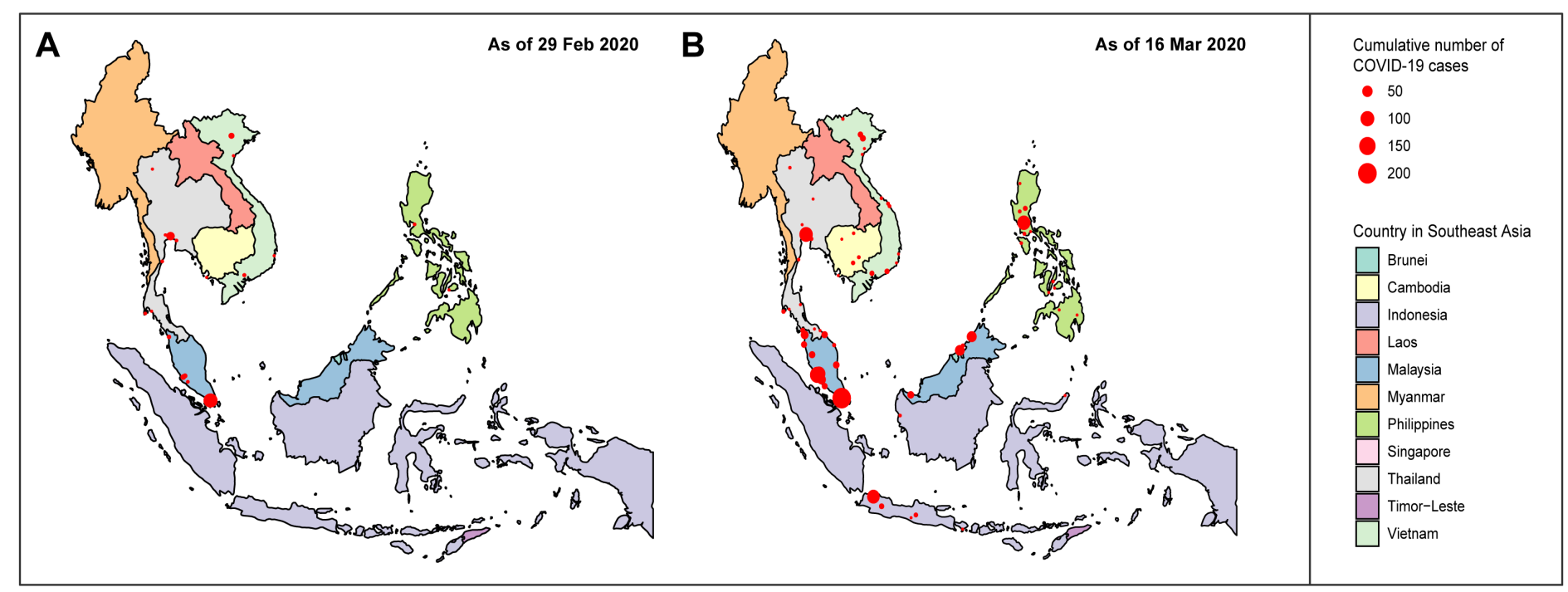

Figure 4 
Spatial distribution of COVID-19 cases in Southeast Asia as of (A) 29 February and (B) 16 March 2020.

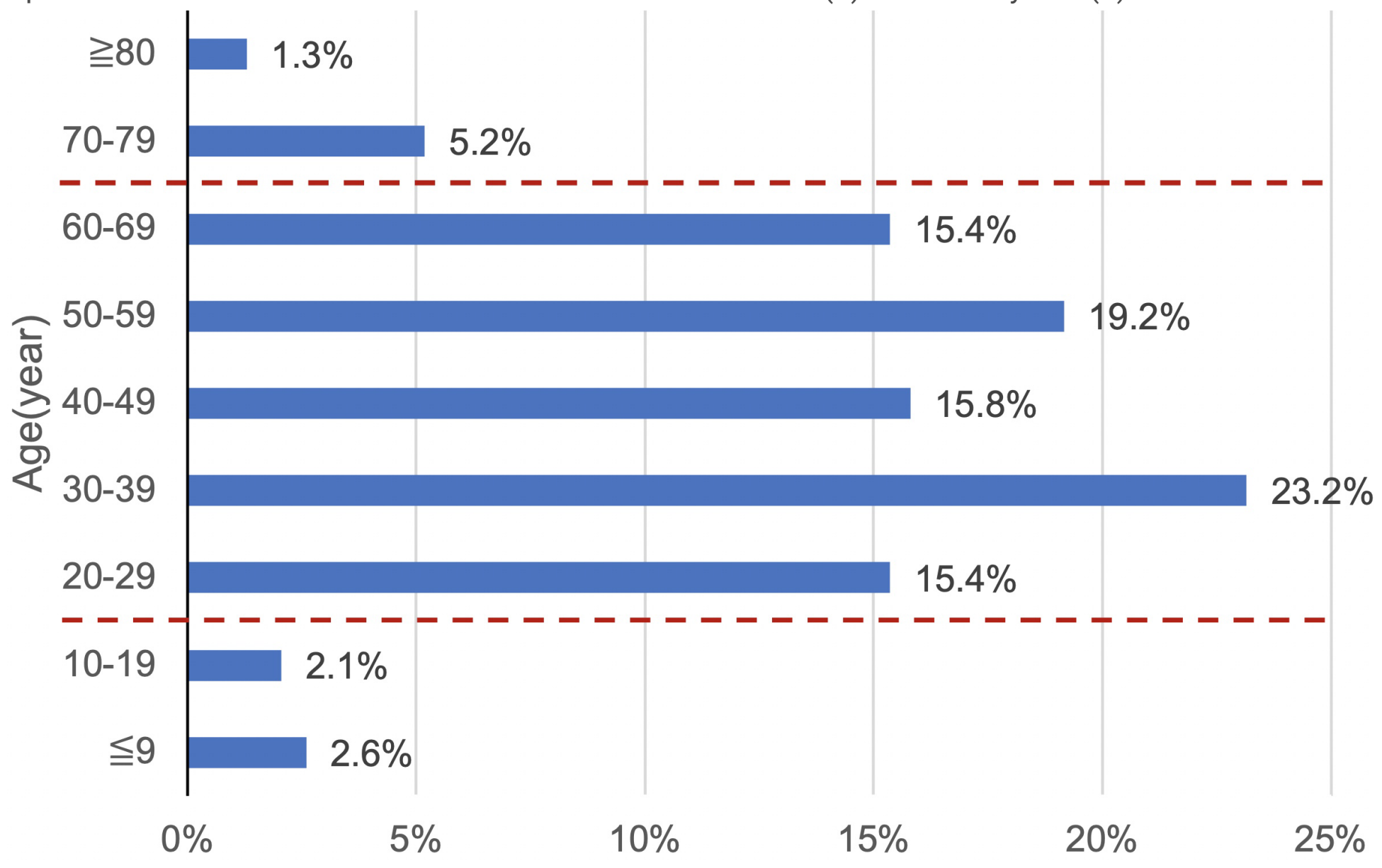

\section{Figure 5}

Age distribution of confirmed COVID-19 cases in Southeast Asia. Red dashed lines: upper and lower limits of the age group in which $88.9 \%$ of cases were concentrated.

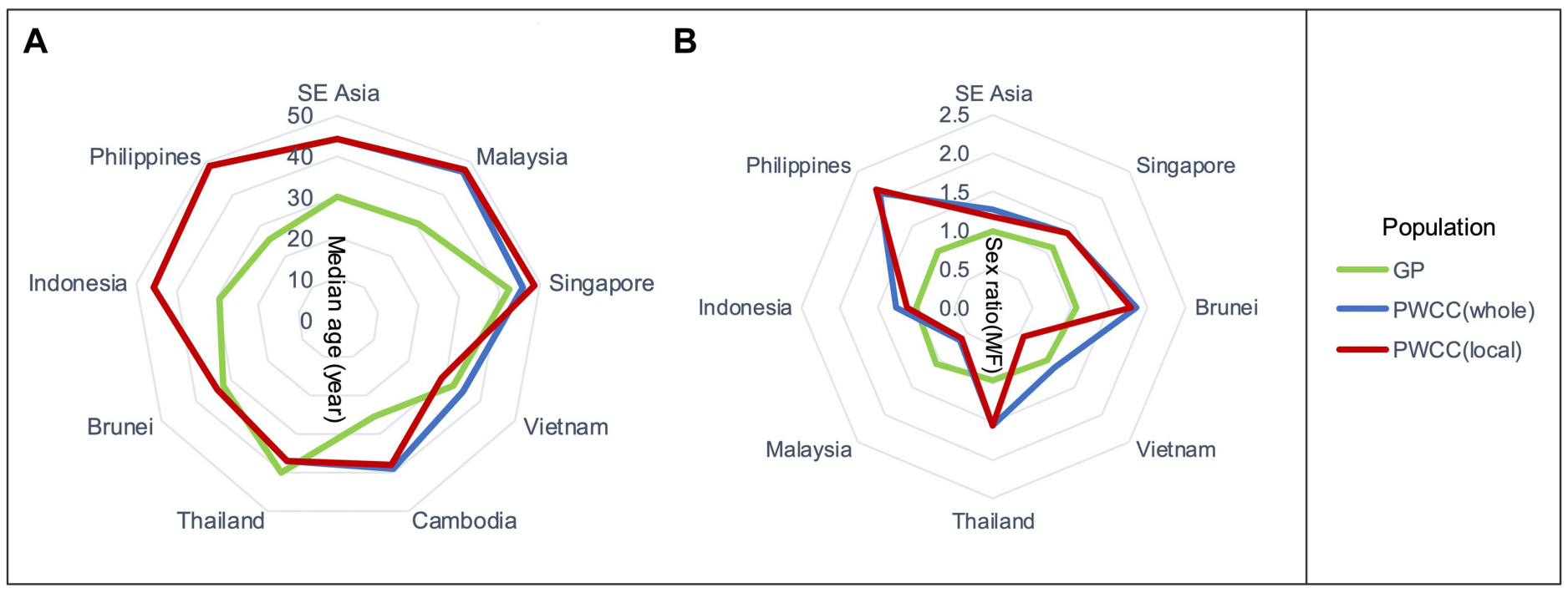

Figure 6 
Demographic constitution of confirmed COVID-19 cases in Southeast Asia (SE Asia) in terms of (A) age and (B) sex. GP: general population; PWCC (whole): patients with confirmed COVID-19 as a whole, whether of local nationality or not; PWCC (local): patients with confirmed COVID-19 who were of local nationality. 